\title{
The 'PUCE CAFE' Project: the First 15K Coffee Microarray, a New Tool for Discovering Candidate Genes correlated to Agronomic and Quality Traits
}

\author{
Isabelle Privat ${ }^{*}$, Amélie Bardil ${ }^{4}$, Aureliano Bombarely Gomez ${ }^{3}$, Dany Severac ${ }^{2}$, Christelle Dantec ${ }^{2}$, Ivanna Fuentes ${ }^{2}$, \\ Lukas Mueller ${ }^{3}$, Thierry Joët ${ }^{5}$, David Pot ${ }^{6}$, Séverine Foucrier ${ }^{1}$, Stéphane Dussert ${ }^{5}$, Thierry Leroy ${ }^{5}$, Laurent Journot ${ }^{2}$, \\ Alexandre de Kochko ${ }^{5}$, Claudine Campa ${ }^{5}$, Marie-Christine Combes ${ }^{4}$, Philippe Lashermes ${ }^{4}$, Benoit Bertrand ${ }^{4}$
}

\begin{abstract}
Background: Understanding the genetic elements that contribute to key aspects of coffee biology will have an impact on future agronomical improvements for this economically important tree. During the past years, EST collections were generated in Coffee, opening the possibility to create new tools for functional genomics.

Results: The "PUCE CAFE" Project, organized by the scientific consortium NESTLE/IRD/CIRAD, has developed an oligo-based microarray using 15,721 unigenes derived from published coffee EST sequences mostly obtained from different stages of fruit development and leaves in Coffea Canephora (Robusta). Hybridizations for two independent experiments served to compare global gene expression profiles in three types of tissue matter (mature beans, leaves and flowers) in C. canephora as well as in the leaves of three different coffee species (C. canephora, C. eugenoides and C. arabica). Microarray construction, statistical analyses and validation by Q-PCR analysis are presented in this study.

Conclusion: We have generated the first $15 \mathrm{~K}$ coffee array during this PUCE CAFE project, granted by Génoplante (the French consortium for plant genomics). This new tool will help study functional genomics in a wide range of experiments on various plant tissues, such as analyzing bean maturation or resistance to pathogens or drought. Furthermore, the use of this array has proven to be valid in different coffee species (diploid or tetraploid), drastically enlarging its impact for high-throughput gene expression in the community of coffee research.
\end{abstract}

\section{Background}

In recent years, microarray technology has demonstrated the power of the high-throughput study of gene expression in unravelling key processes in plant biology [1-3]. Microarrays have become especially relevant for species where little genome information is available and where intensive laboratory work is necessary to gain insight into a particular biological process, as well as to identify candidate target genes for future breeding programs $[4,5]$.

The genus Coffea (Rubiaceae family) encompasses approximately 100 species, all of which are native to the African continent, Madagascar and the Mascarene

\footnotetext{
* Correspondence: isabelle.privat@rdto.nestle.com

${ }^{1}$ Nestlé R\&D Tours, 101 Avenue Gustave Eiffel, Notre Dame d'Oé, BP 49716, 37097 Tours Cedex 2, France

Full list of author information is available at the end of the article
}

Islands [6]. Two of these species Coffea canephora (robusta) and Coffea arabica, are widely cultivated for the production of coffee beverages. The former is diploid $(2 n=2 x=22)$ and allogamous, the latter, allotetraploid $(2 \mathrm{n}=4 \times=44)$ and preferentially autogamous. Approximately $60 \%$ of the world coffee production comes from C. arabica versus $40 \%$ for C. canephora. In terms of cup quality, consumers appreciate C. arabica (Arabica) more due to its taste, which is less bitter and more flavourful compared with C. canephora (Robusta). While it is not widely known, coffee is one of the most valuable international exchange commodities in agricultural trade. This is reflected in the fact that raw coffee values rank fourth on the international stock market only after wheat, sugar, and soya [7]. Furthermore, over 25 million people worldwide are linked to coffee cultivation and processing. Despite these economic aspects,

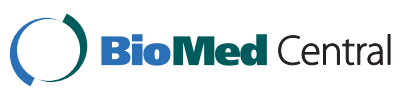

() 2011 Privat et al; licensee BioMed Central Ltd. This is an Open Access article distributed under the terms of the Creative Commons Attribution License (http://creativecommons.org/licenses/by/2.0), which permits unrestricted use, distribution, and reproduction in any medium, provided the original work is properly cited. 
coffee research suffers from a lack of both scientific and financial investment. Also, coffee is a perennial plant which only begins to bear seed after about five years, which makes genetic studies more complicated and time-consuming. While some genomic information is publicly available for coffee (e.g., an expressed sequence tag (EST) database), it lags far behind what is available for many other agricultural species. As a result, coffee researchers have only limited access to the plethora of genomic resources available for most major crop species.

During the past few years, aiming to develop genomic tools to assist future coffee research, various scientific groups have produced large scale sets of Coffea EST sequences. However, the number of publicly available ESTs remains low because many of the sequences discovered fall under the domain of private property and are not published. At the time when the PUCE CAFE Project began, two large coffee EST databases were available: the NESTLE/Cornell and IRD databases with respectively 62,877 and 8782 sequences. Those sequences were mainly cDNA derived from leaves, fruit (whole cherries), pericarp and beans at different stages of maturation in Coffea canephora (robusta) $[8,9]$. The research aimed to catalogue as many genes as possible which are involved in the bean-filling period of fruit development in order to better understand the final composition of the beans which constitute the commercial product. The purpose of the PUCE CAFE Project was to develop a long oligonucleotide array based on available sequences and thus to use this new tool to perform large-scale transcriptomic analyses in different areas such as bean/fruit development, polyploidy or drought resistance in Coffea canephora or Coffea arabica. To assess its utility, we ran a comparison between three different tissues, i.e. mature beans, flowers and fully-expanded leaves, in Coffea arabica in order to catalogue genes specifically expressed in each tissue. We analyzed in particular the genes involved in fatty acid synthesis and storage proteins and compared our results with those in recent publications on Coffea [10] and also with exalbuminous bean species. Then we tested the usability of our 15k microarray for three coffee species.

\section{Methods}

\section{The Coffee Gene Assembly (Build II)}

To create the SGN Coffee Unigene Build II http://solgenomics.net/, 71,659 EST (Expressed Sequence Tag) chromatograms were processed from the following C. canephora sequence libraries: cccl (coffee leaf, 11,655 chromatograms), cccp (coffee pericarp, 10,849 chromatograms), cccs18w (coffee early-stage bean, 1,972 chromatograms), cccs30w (coffee middle-stage bean, 15,318 chromatograms), cccs $42 \mathrm{w}$ (coffee late-stage bean, 42 weeks after pollination, 469 chromatograms), cccs $46 \mathrm{w}$ (coffee late-stage bean, 46 weeks after pollination, 10,907 chromatograms), cccwc22w (coffee early-stage whole fruit, 11,660 chromatograms), irdccf (IRD coffee cherry in various developmental stages, 5,089 chromatograms), irdccl (IRD, young leaves, 3,693 chromatograms) and nDav1 (Nestle Dav1, 47 chromatograms), using PHRED software http://www.phrap.org/phredphrapconsed.html[11]. The sequences were processed to remove vector, adaptors and low complexity sequences using an SGN-developed Perl script). Chimeric sequences were screened by processing the BLAST results [12] using Arabidopsis thaliana ftp://ftp. arabidopsis.org/home/tair/Sequences/ as reference dataset and a SGN Perl script. A total of 55,539 sequences passed the filter tests and were used in the assembly. The unigene assembly was created in two steps. First, using a self-BLAST and an SGN Perl script (precluster. $\mathrm{pl}$ ), we implemented a pre-clustering phase of the EST sequences with a minimum identity percentage of $90 \%$ and a minimum alignment length of $30 \mathrm{bp}$. Secondly, we used CAP3 software http://seq.cs.iastate.edu/[13] for each cluster with the following parameters: -e 5000 -p 90 -d 10,000 -b 60. The -e, -d and -b options were set so that the assembler would disregard them or minimize their effect. The -p option increased the sequence identity necessary with overlaps to 90 from a default of 75 , and thus was found to be lacking in stringency.

Concerning unigene annotations, we first made a homology search using the BLAST program against GenBank ftp://ftp.ncbi.nih.gov/genbank/ and Arabidopsis thaliana ftp://ftp.arabidopsis.org/home/tair/Sequences/ datasets, setting an e-value of $1 \mathrm{e}-10$ as the cutoff value. Secondly, we implemented a prediction of protein sequences based on unigene sequences using ESTScan software http://estscan.sourceforge.net/[14] and an SGN Perl script (longest6frame.pl), which simply determines the longest open reading frame and translates it into a protein sequence. Thirdly, we set up a protein domain homology search on predicted protein sequences using InterProscan software http://www.ebi.ac.uk/Tools/InterProScan/[15]. All the information concerning the different scripts used to perform EST assembly are available on https://github.com/solgenomics/sgn-home/tree/ master/aure/scripts/old_sgn_transcript/.

\section{Long Oligonucleotide Microarray Design and Synthesis}

The Coffea canephora long oligonucleotide set was designed and synthesized by Operon (Cologne, Germany) based on the SGN Coffee Build II $(15,721$ unigenes; http://solgenomics.net/). An amino linker was attached to the 5'-end of each oligonucleotide. The oligonucleotides, selected to limit secondary structure, 
have a melting temperature of $67 \pm 3^{\circ} \mathrm{C}$, length $65 \pm 5$ bases, GC content $43 \pm 5 \%$. More than $98 \%$ of the oligonucleotides were within 1000 bases from the 3'-end of the available gene sequence. For 195 unigenes, no adequate oligonucleotide could be designed and therefore correspond to "missing genes" (Additional File 1). BLAST alignments were performed to identify oligonucleotides that could cross-hybridize with other sequences of the SGN Coffee Build II. Finally out of 15,522 oligonucleotides designed, there are 371 oligonucleotides which have $>70 \%$ of overall identity to another unigene and have a contiguous identical length of over 20 nt common to another unigene (Additional File 2).

\section{Plant Material and RNA Extraction}

In a first experiment (Experiment 1), we compared three tissues, namely fully-expanded leaves, open flowers and mature beans. They were collected from C. arabica L. cv. Caturra T 2308 grown in greenhouse conditions in Tours, France.

In a second experiment (Experiment 2), we compared fully-mature leaves of three species (namely C. arabica, C. canephora and C. eugenioïdes) to determine if our microarray could be used for different coffea species. $C$. arabica was represented by the $c v$. 'Java' issued from the Arabica woodland Ethiopan pool and by one genotype representing the Arabic-cultivated pool. C. canephora was represented by the $c v$. 'Nemaya' derived from the cross of two Congolese genotypes. Finally, C. eugenioides was represented by several genotypes, collected in Kenya at the Mount Elgon. The coffee seedlings were grown in a greenhouse with natural daylight and a constant temperature of $24^{\circ} \mathrm{C}$ and watered as necessary. After 120 days, the plants were transferred for an additional 60 days to a phytotron chamber (CRYONEXT, France, model RTH 1200L). The standard conditions in the phytotron were $12-\mathrm{hr}$ light $\left(600 \mu \mathrm{mol} . \mathrm{m}^{-2} \mathrm{~s}^{-1}, 26^{\circ} \mathrm{C}\right)$, 12 -hr dark $\left(22^{\circ} \mathrm{C}\right)$, with $80 \%$ to $99 \%$ relative humidity. In each growth chamber three plants for each species were cultivated. Each plant represented one replicate. Two fully-developed leaves were collected from each plant (i.e. two leaves/replicate) at noon (6-8 hours after lights on) and then flash-frozen in liquid nitrogen.

Tissues were ground into a powder and total RNA was extracted using the RNeasy Plant Mini Kit (Qiagen; Valencia, Cal., USA), then treated with DNase following the manufacturer's instructions. Total RNAs were finally eluted from the columns with RNase-free water $(2 \times 30$ $\mu \mathrm{L})$. For each tissue, three independent RNA extractions were performed. All RNA samples were analyzed by formaldehyde agarose gel electrophoresis to assess their integrity. To test for contamination by polyphenols, carbohydrates and proteins, a NanoDrop ND-1000 spectrophotometer (NanoDrop Technologies; Wilmington,
Delaware, USA) was used. Only RNA samples with OD $260 / 280>1.8$ and $\mathrm{OD} 260 / 230>2$ were used for further analysis.

\section{RNA Labelling}

For the preparation of the labelled Cy3- and Cy5- aRNA target, one microgram of the total RNA samples were amplified and labelled using the Amino Allyl Message Amp II aRNA Amplification Kit (Ambion; Austin, Texas, USA), according to the manufacturer's instructions.

\section{Microarray Printing}

The synthesized oligonucleotides were arranged in 384well plates, and dissolved at $20 \mu \mathrm{M}$ in a phosphate buffer (150 mM, pH 8.5). The oligonucleotide probes were printed on reflective epoxysilane-coated slides (Amplislide, Genewave, Ecole Polytechnique, France) using a Lucidea Array printer (GE HealthCare, St. Catharines, Ontario). The oligonucleotides library also included sets of positive and negative control points that were used for verifying, for example, the quality of the microarray and mRNA, the sensitivity and linearity of the signal, or the consistency of the assay. In addition, the expected dye ratios were determined and the differences in signal intensities due to the differences in dye incorporation and quantum yield were estimated.

\section{Hybridization}

Prior to hybridization, oligonucleotides were cross linked to the slides by UV irradiation at $100 \mathrm{mj}$ and the excess was removed from the arrays by washing them twice in one minute in $0.2 \%$ sodium dodecyl sulphate (SDS). Arrays were then washed twice in distilled water. The two labelled aRNA were added to Microarray Hybridization Buffer Version 2 (GE HealthCare, St Catharines, Ontario) in a final concentration of $50 \%$ formamide, denaturated at $95^{\circ} \mathrm{C}$ for three minutes and applied to the microarrays in individual chambers of an automated slide processor (GE HealthCare, St Catharines, Ontario). Hybridization was carried out at $37^{\circ} \mathrm{C}$ for 12 hours. Hybridized slides were washed at $37^{\circ} \mathrm{C}$ successively with $1 \times$ Saline Sodium Citrate, $0.2 \%$ SDS for 10 minutes, twice with $0.1 \times$ SSC, $0.2 \%$ SDS for 10 min, with $0.1 \times$ SSC for one minute and with isopropanol before air drying.

\section{Data Acquisition}

Microarrays were immediately scanned at $10 \mu \mathrm{m}$ resolution in both Cy3 and Cy5 channels with GenePix 4200AL Scanner (Molecular Devices, Silicon Valley, Cal., USA) with variable photo multiplier tube (PMT) settings to obtain maximal signal intensities with $<0.1 \%$ probe saturation. ArrayVision ${ }^{\circledR}$ software (GE HealthCare, 
St Catharines, Ontario) was used for feature extraction. Spots with high local background or contamination fluorescence were flagged manually. A local background was calculated for each spot as the median values of the fluorescence intensities of four squares surrounding the spot.

\section{Real-time PCR}

We carried out reverse transcription of total RNA using random hexamer oligonucleotides and SuperScript II Kit (Invitrogen, Carlsbad, Cal., USA) according to the manufacturer's instructions. Real-time PCR was performed on a LightCycler ${ }^{\circledR} 480$ equipped with a 384-well block using the LightCycler ${ }^{\circledR} 480$ SYBR Green I Master Mix (Roche Diagnostics, Indianapolis, Ind., USA) according to the manufacturer's instructions. The primer sequences used for the determination of gene expression levels are given in Additional File 3. The selection of appropriate housekeeping genes was performed using geNorm [16]. The level of expression of each gene $\mathrm{X}$ was normalized to the geometric mean of the expression levels of 3 reference genes (Spermidine synthase 1, Cyclophilin and Actin-11), according to the formula

$$
\frac{X}{\sqrt[3]{R 1 \times R 2 \times R 3}}=2^{\left(C t(X)-\left(\frac{C t(R 1)+C t(R 2)+C t(R 3)}{3}\right)\right)}
$$

where $\mathrm{Ct}$ is the threshold cycle and R1, R2, R3 are the 3 reference genes.

Additional information concerning the Q-PCR experiment can be found in the MIQE document (Minimum Information for Publication of Quantitative Real-Time PCR Experiments) (See Additional File 4).

\section{Experimental Design and Data Analysis}

For the first experiment, for qRT-PCR as for microarray, three biological replicates were made for each tissue analyzed (i.e. leaves, flowers and mature beans). The following comparisons were made: Bean-Flower, Leaf-Flower and Leaf-Bean. In all, we performed microarray analyses on 18 slides [3 (replicates) $\times 2$ (dyes) $\times 3$ (organs)]. For qRT-PCR we performed 3 technical replicates $\times 3$ biological replicates $\times 3$ organs for 108 genes and three reference genes (R1, R2 and R3) for each tissue.

For the second experiment, three biological replicates were done, each of one containing two leaves. In total we used 36 slides [3 (replicates) $\times 2$ (dye) $\times 6$ (comparisons)]. All microarray analyses were performed using Bioconductor http://www.bioconductor.org, the open development software project for the analysis and comprehension of genomic data.

\section{Preprocessing}

A quality analysis was made by generating image plots (MA-plots, boxplot, visualization of the array).
No background correction was performed. Few spots were flagged and controls were removed for the normalization. Loess normalization was performed for each microarray to correct the dye effect and technical bias. Then the microarray data were filtered keeping the spot intensity above a median of 90 percent of the control spots (Empty/Negative Control - NC) plus twice the deviation standard, applied to both channels (Red and Green).

\section{Statistical Analysis}

Two tests of differential expression were conducted simultaneously: the first test fit a linear model for the expression data for each gene by using the Limma Package (Linear Models for Microarray Data) [17], the second test SAM used repeated permutations of the data to determine significant genes [18]. It was conducted with the Siggenes package from Bioconductor. Multiple testing adjustments were performed by using a false discovery rate approach [19]. These two analyses allowed us to rank significantly expressed genes. The Bioarray Software Environment (BASE - [20]) (local installation: http://baseprod.igf.cnrs.fr/index.phtml) was used to visualize the differential expression for each gene.

\section{Reproducibility of Biological Replicates and Specificity of the Microarray}

For the first experiment, coefficients of variation were calculated for the mean signal intensity for the 6 slides (i.e, two dyes $\times$ three biological replicates). Using the procedure rank in SAS 9.2 (SAS Institute, Cary, NC), each CV received a rank according to the size of the $\mathrm{CV}$ value. These rank values were then expressed on a 100based scale.

The effect of sample size on the power of statistical tests for different CVs was estimated using the sample size estimate procedure for a two-sample t-test in SAS 9.2 with $\alpha=0.01$, group 1 mean $=1$, and group 2 mean $=1.5$ or 2.5 . The effect of the $\mathrm{CV}$ on the minimally detectable expression ratio (threshold expression ratio) was iteratively estimated for a power of 0.9 and $\alpha=0.01$ using the sample size estimate procedure for a two-sample t-test.

To validate the expression changes found in Microarray Experiment 1, transcript levels of 108 genes and three reference genes were quantified by Q-PCR (with validated primers [10]). Results obtained by both techniques (microarray and Q-PCR) (Additional File 3) were compared by calculating the Pearson correlation coefficients (SAS 9.2). For the microarray, the data input into the correlation analysis was the $\log _{2}$ ratio value of the weighted average for each gene on the composite array representing all replicates. For qRT-PCR, we used the mean $\log _{2}$ ratio value representing all replicate plants. 
All correlation analyses carried an alpha value of 0.01 and were performed using SAS 9.2.

For the second experiment, background noise was defined from the intensity of "negative" controls (NC). The median of the negative controls was calculated. A gene was considered expressed if the intensity exceeded twice the standard deviation. If a gene is significantly expressed during a comparison (6 hybridizations), its signal should be superior to the highest background noise in each hybridization (maximum 6 times). We chose to fix this threshold to 5 , and for each species we screened the number of genes that reached or surpassed this value (Additional File 5).

A hierarchical tree-clustering support method was performed using TMeV 4.0 software from TIGR. The Euclidean distance was used as a measure of similarity or distance between hybridizations. As a rule, the Average-Link Method was used for linking clusters. With this method, distance calculations are based on pairs of clusters: taking the average between the distance of objects from the first cluster and of objects from the second cluster. The averages are performed for all pairs to determine the actual distance between clusters.

Venn diagrams were generated using the online Venny tool http://bioinfogp.cnb.csic.es/tools/venny/index.html.

\section{Gene Ontology Functional Enrichment Analysis}

Computational annotation was also performed using Blast2GO software v2.4.4. (http://www.blast2go.org website) [21]. The annotation step was performed using the BlastX algorithm, the NCBI $\mathrm{nr}$ database and a Blast expectation value threshold of $1 \mathrm{E}-3$. The Blast $2 \mathrm{GO}$ tool was then used to obtain GO information from retrieved database matches. Mapping of all sequences was performed using default parameters. An InterPro Scan was also performed to find functional patterns and related GO terms by using the specific tool implemented in the Blast2GO software with the default parameters. Finally, an enrichment analysis was completed for the sets of up-regulated unigenes in each of the three tissues studied using the corresponding Blast2GO module, which is based on Fisher's Exact Test and FDR statistics. Additional File 6 shows GO terms showing a significantly higher or lower frequency in tissue-specific unigene sets in comparison with the full set of unigenes of the PUCE CAFE array.

\section{Availability of the Microarray Data}

Microarray data are publicly available at http://www. ncbi.nlm.nih.gov/geo/. The GEO accession number is GSE24754 for Experiment 1 and GSE24682 for Experiment 2. The array is referenced as GPL10928.

\section{Results and Discussion Chip Quality}

To estimate the quality of the work performed by the MGX platform (Montpellier, France), one validation experiment (Experiment 1) was implemented. Expression was compared in three tissues, namely fullyexpanded leaves (L), open flowers (F) and mature bean (B) in Coffea Arabica. Three biological replicates were used for each tissue. The following comparisons were studied: Bean-Flower, Leaf-Flower and Leaf-Bean.

Raw quantification and background noise values were represented for each chip (data not shown). Visually, the flags indicated invalidated spots. In this way it was possible to visualize whether there were any particular artefacts on a slide, due for example to washing impurities or to the presence of dust. The distribution of raw intensities, background noise and log-ratios were uniform. Very few spots were flagged (Table 1) and background noise was low and virtually constant when signal intensity increased (Figure 1) indicating that the chips were of very good quality.

\section{Signal Distribution for each Hybridization and Data Standardization}

Gene expression was compared as a function of the dye (Cy3 or $\mathrm{Cy} 5)$. Background noise was defined from the intensity of "negative" controls (NC). The median of the negative controls was calculated. A gene was considered expressed if the intensity exceeded twice the standard deviation. We compared efficiency for Cy3 and for Cy5 incorporation. The dye bias was greater for low-value signals. Of 15,998 genes, $40.76 \%$ were always significantly expressed compared to background noise for the red dye and $44.70 \%$ for the green dye. This bias was largely corrected by standardizing the data using the Loess regression method.

After standardization, the curves for the $\mathrm{Cy} 3$ and $\mathrm{Cy} 5$ signals were superposed upon the density graphs (Figure 2). The MA-plot scatterplots did not display any striking differences as most of the technical biases were reduced by using automated protocol as well as specific slides for reducing differences due to dye incorporation.

Box-plots of both pre- and post-normalization (Figure $3 \mathrm{~A}$ and $3 \mathrm{~B}$ ) confirmed that our data were successfully

Table 1 In Experiment 1, for each comparison (six slides) 16,512 spots were examined

\begin{tabular}{cc}
\hline Comparisons & $\begin{array}{c}\text { Number of Spots invalidated by visual examination } \\
\text { (Flags) }\end{array}$ \\
\hline Leaf/bean & 16 \\
Leaf/flower & 34 \\
Bean/flower & 7 \\
\hline
\end{tabular}

The number of genes invalidated by visual examination is indicated. 

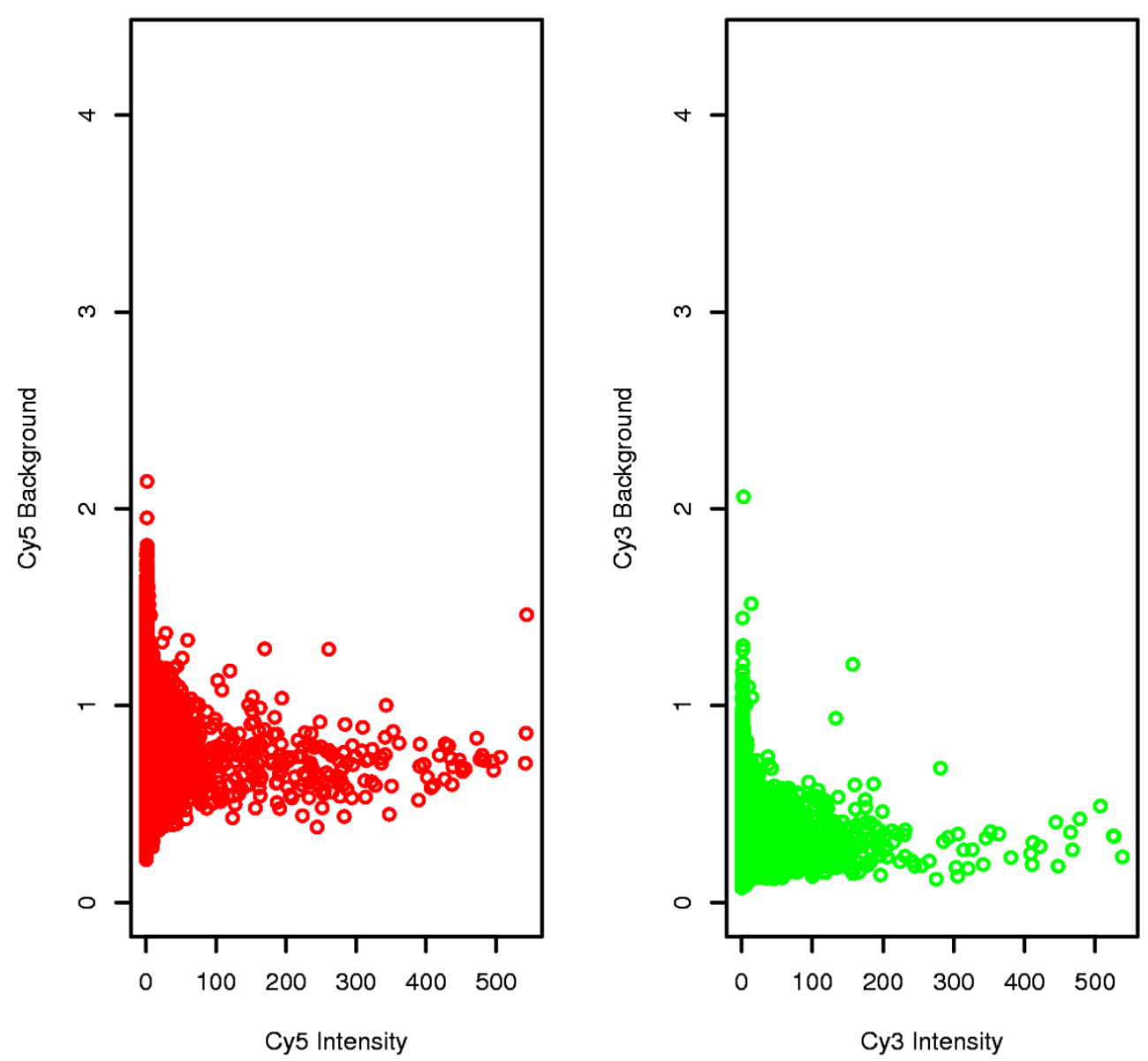

Figure 1 Plots for each Channel of Background Intensity versus Intensity. The background is uncorrelated and does not increase along with the intensity. Flower vs. leaf comparison data are presented here.

normalized. Data quality was assessed by comparing the signal intensity data from each array to that obtained from the technical or biological replicates. Pearson correlation between replicates was calculated for every gene in all the arrays, resulting in a very high correlation level, with a coefficient of $>0.89$ for every independent experiment in a pairwise comparison (Figure 3D). This high coefficient is indicative of the precision level in which the microarray is able to process transcriptomic data reliability.

A support tree-clustering method with bootstrapping using expression data was performed to statistically validate the tool. Figure $3 \mathrm{C}$ showed a high level of similarity between the replicates.

\section{Reproducibility of Biological Replicates}

The variability between expression profiles derived from the two dyes and the three biological replicates was estimated. For each experiment we calculated the coefficient of variation $(\mathrm{CV})$ for the mean signal intensities for six slides (i.e. 2 dyes $\times 3$ biological replicates). Ninety percent of the spots on the arrays could be determined with a CV of less than $42 \%$ (Table 2). These values are consistent with those obtained previously [22]. The CV (\%) was then plotted against the relative rank of the CV (Additional file 7 ). Based on this estimated variance, a power analysis was performed for a two-sample t- test (Additional file 8).

With a CV of $35 \%$, a sample size of six slides can detect a 2-fold change in gene expression with a power of $90 \%$, with a Type I error rate of $10 \%$. The detection of 1.5 -fold changes with a Type I error rate of 0.1 , a power of $90 \%$ and sample size of six slides requires the CV to be below 20\%. We therefore concluded that a sample size of six slides would allow acceptable control of both Type I and Type II errors.

\section{Analysis of Differential Expressions}

Two statistical analyses were performed on normalized data from Experiments 1 and 2, one by the Limma 


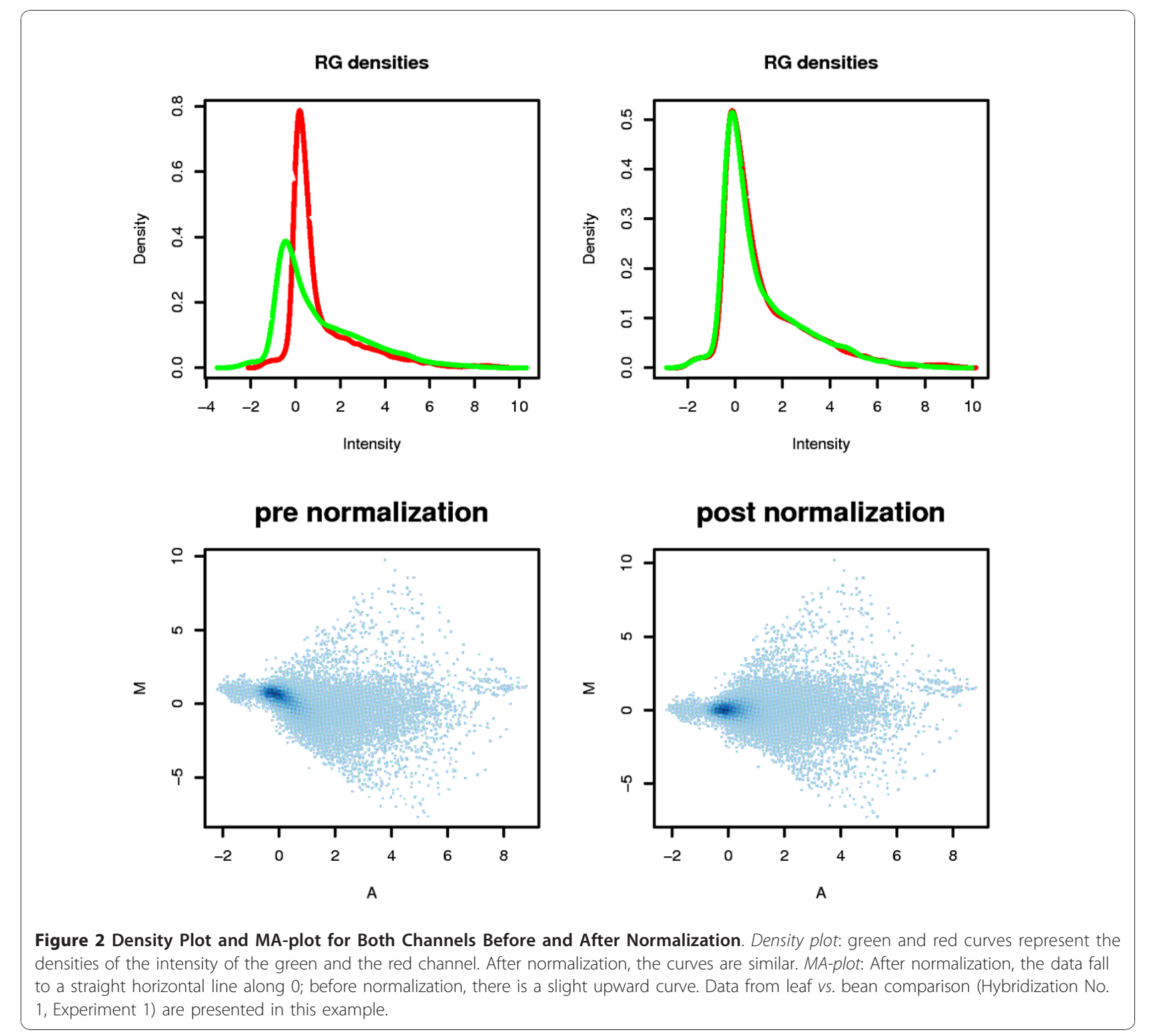

Method (linear model), the other by the SAM Method (significance analysis for microarray) with two thresholds $\mathrm{P}=0.01$ and $\mathrm{P}=0.05$. For each comparison, lists of differentially-expressed genes were generated. One list was derived from the "Limma" analysis, the other from the "SAM" analysis. The files are available on BASE http://baseprod.igf.cnrs.fr/index.phtml. The Limma Method was more restrictive than the SAM Method, so the number of genes determined as being significantly differentially expressed was therefore smaller. Nevertheless, the totality of these genes was also detected by the SAM Method in the three comparisons conducted. These genes were therefore validated by two independent methods (results not shown). The lists resulting from the SAM 0.01 analysis were used to compare differentially-expressed genes for each comparison (Figure 4 for Experiment 1 and Figure 5 for Experiment 2). The lists resulting from the Limma $P=0.01$ analysis was used to identify over-expressed genes in one specific tissue (bean flower or leaf) (Figure 6 Additional file 9).

\section{Comparison of the Three Tissues (Mature Bean, Flower, Leaf)}

The number of genes that were differential and significantly expressed when comparing different tissues was between 9,021 and 9,999 genes (Figure 4), i.e. between $58 \%$ and $64 \%$ of genes spotted on the array. For each comparison, the percentages of up-regulated genes 


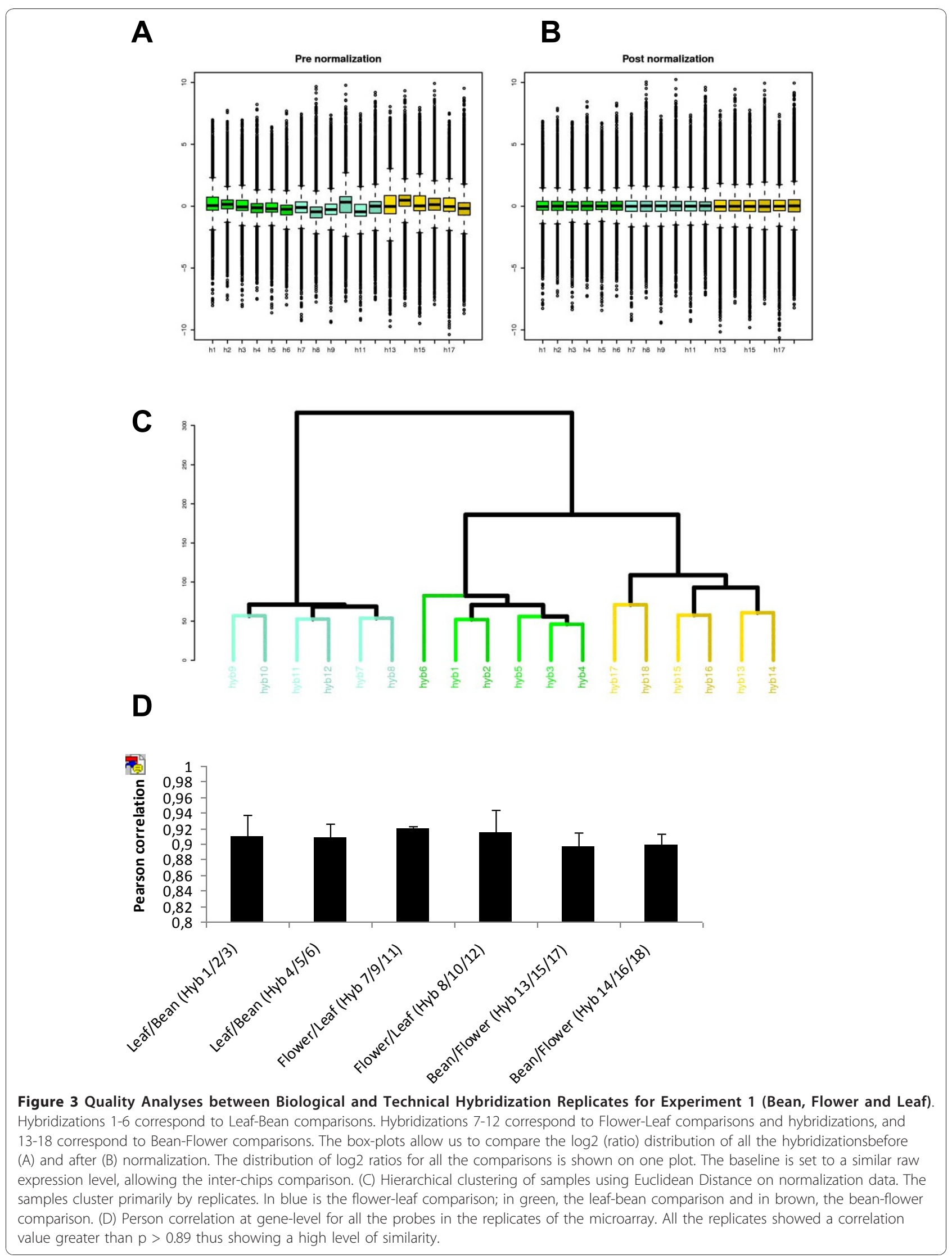


Table 2 Mean, Median and P90 of the Coefficients of Variation (CV) of the Fluorescence Signal Intensity

\begin{tabular}{ccccc}
\hline Comparison & Tissue & Mean & Median & P90 \\
\hline Leaf/bean & Leaf & 27.31 & 24.15 & 41.58 \\
& Bean & 25.66 & 21.82 & 38.93 \\
Leaf/flower & Leaf & 23.38 & 21.76 & 35.68 \\
& Flower & 22.92 & 20.46 & 32.59 \\
Bean/flower & Bean & 23.28 & 20.58 & 37.56 \\
& Flower & 19.90 & 17.96 & 32.26
\end{tabular}

varied between 30 and 33\%. The three tissues therefore behaved globally in an identical manner.

\section{Correlation between qRT-PCR and Microarray Results for} 111 Genes

Consistent with the previous results [23], fold change results determined by $\mathrm{qRT}$-PCR were significantly greater than fold change assessed for the same genes by microarray analysis. Correlations for the data sets (i.e., bean/flower, flower/leaf, leaf/bean) ranged from 0.78 $0.81(\mathrm{p}<0.01$ ) for the 108 genes analyzed (Additional file 3 ). The direction of change was similar for both qRT-PCR and microarray for 70-75\% of the genes analyzed. Furthermore, when we correlated only the significantly-expressed genes (SAM 0.05) (> 1.5 fold change), the degree of correlation between microarray and qRTPCR results was higher, ranging from 0.85-0.87 ( $\mathrm{p}<$ 0.01 ) for 83 genes. The lack of congruence between both methods for genes exhibiting low levels of variation ( $<1.5$ fold change) has been commonly reported [24]. These results validate the implicit assumption that there is a good correlation between the microarray data and the mRNA levels in the tissue under investigation.

\section{Specifically Over-Expressed Genes in each Tissue (Mature} Bean, Flower, Leaf)

Based on the lists of deregulated genes identified for each comparison (Limma Analysis; $\mathrm{P}=0.01$ ), Venn diagrams were constructed (Figure 6). Over-expressed genes in each specific tissue were so listed (Additional file 9). 1,565 genes were significantly over-expressed in the bean compared to the leaves and flowers (Figure 6A and Additional file 9). Likewise, 220 "flower-specific" (Figure 6B) and 176 "leaf-specific" (Figure 6C) genes were identified.

The number of genes which are over-expressed in the bean is significantly higher than those identified in flower and leaf. This observation is quite normal since the PUCE CAFE array is mainly based on genes expressed in the grain during fruit maturation.

This analysis is quite interesting and clearly identified the genes involved in different metabolic pathways specific to each organ. In order to shed light onto the processes involved under the conditions studied, we enriched the Gene Ontology (GO terms) among upregulated genes in the three different organs. Additional file 6 shows GO terms showing a significantly higher or lower frequency in tissue-specific unigene sets in comparison with the full set of unigenes of the PUCE CAFE array.

Validity of Microarray Results related to Biosynthesis Pathways of Lipids or Storage Proteins

To test the accuracy of the results obtained with the $15 \mathrm{k}$ coffee microarray a bit further, we compared expression

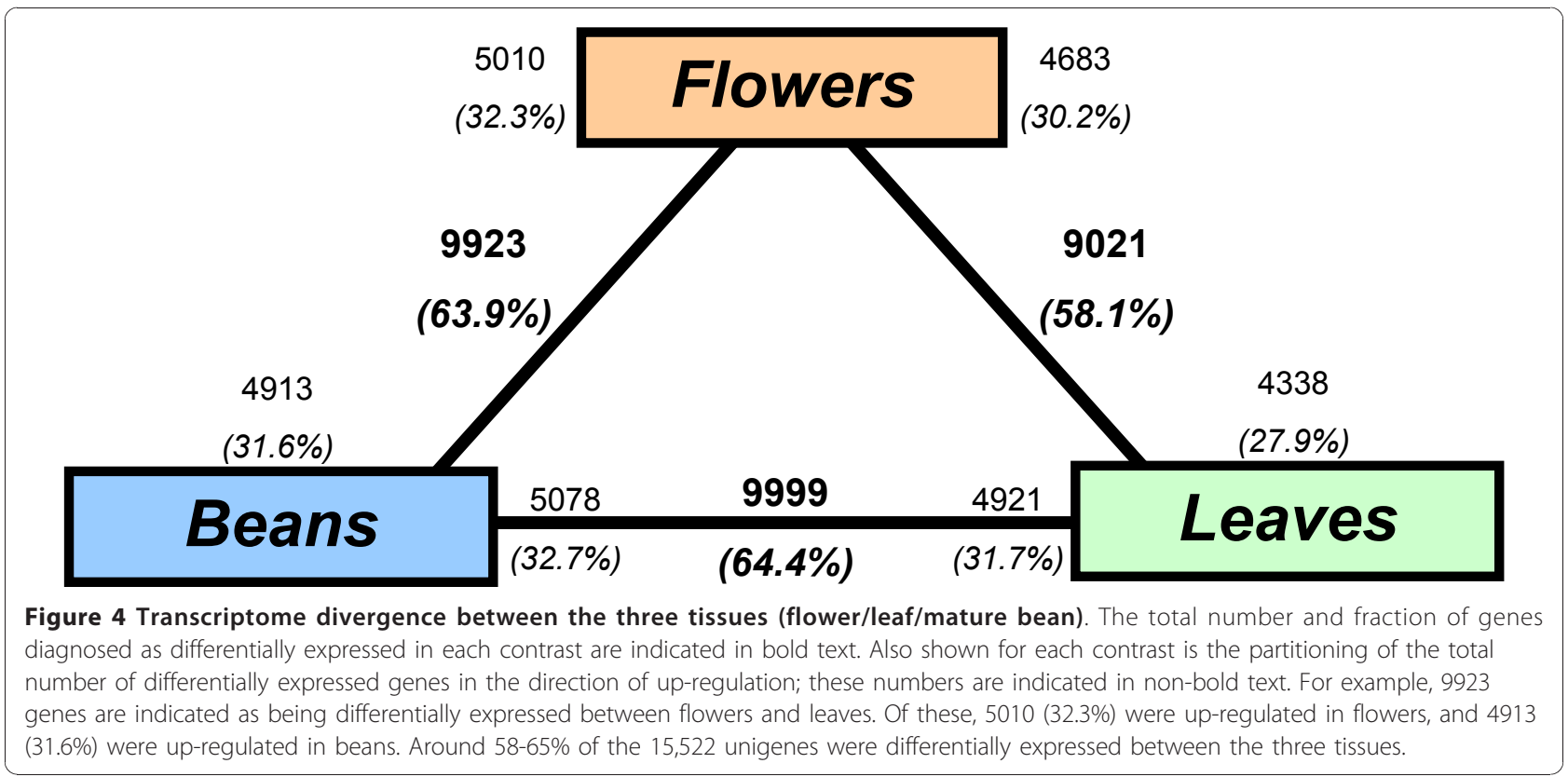




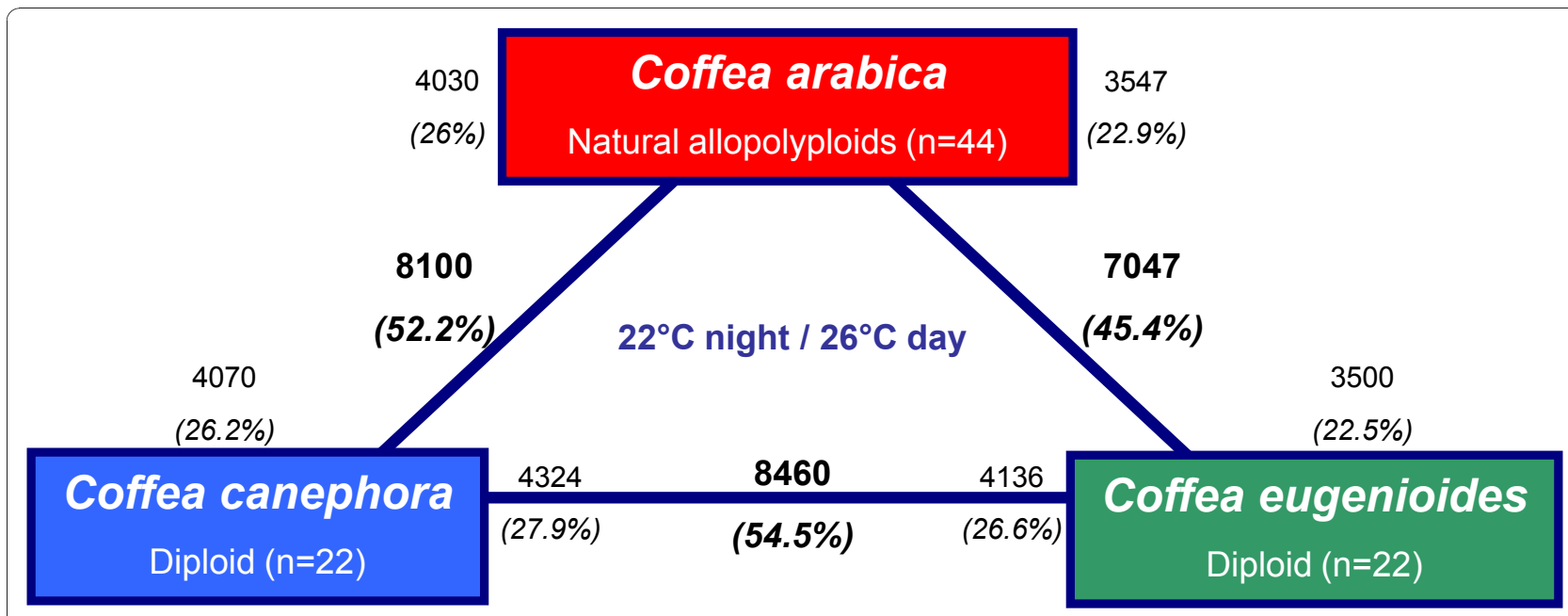

Figure 5 Transcriptome divergence between $\boldsymbol{C}$. arabica, $\boldsymbol{C}$. canephora and $\boldsymbol{C}$. eugenoides. Bold text indicates the total number and fraction of genes that were defined as differentially expressed between each comparison. Non-bold text indicates the total number and fraction of genes that were in the direction of up-regulation. For example, 8100 (52.2\%) genes were indicated as being differentially expressed between C. canephora and C. arabica. Of these, 4030 (26\%) were up-regulated in C. arabica, and 4070 (26.2\%) were up-regulated in C. canephora.

patterns of a few genes involved in well-characterized biosynthetic pathways of lipids or storage proteins with those described in studies on Coffea and model plants. As storage tissue, the mature endosperm accumulates nutrient reserves (mainly cell-wall polysaccharides, sucrose, proteins and oils) which are mobilized by the embryo during germination and seedling growth. As expected, most of the genes involved in the accumulation of these storage compounds displayed enhanced transcriptional activity in the bean compared to leaves and flowers.

\section{Study of Different Genes Involved in the Biosynthesis of Lipids}

In coffee leaves, linolenic acid (18:3) is the predominant FA (fatty acid) [25], whereas it represents only a small percentage of the total FA in beans [26]. Accordingly, the gene encoding the enzyme involved in converting linoleic acid to linolenic acid ( $\omega-3$ desaturase, FAD 8 ) was significantly over-expressed in leaves compared to beans (Table 3). Similarly, although waxes can be detected in beans and flowers, these compounds predominantly accumulate in leaves. The first step of wax biosynthesis involves a $\beta$-Ketoacyl-CoA Synthase (KCS) activity, which initiates the biosynthesis of a very-long chain of fatty acids specific to waxes. Again, the microarray showed evidence of a higher KCS gene expression in leaves compared to other tissues (Table 3).

The acyl-ACP thioesterase (encoded by the Fat B gene) was described as the control point of the remarkably high palmitic acid content of Arabidopsis flowers in comparison with other organs [27]. A similar expression pattern was observed for the putative FatB gene in coffee, suggesting that the coffee flower could also be highly rich in this fatty acid. Finally, since coffee flowers are well known for their jasmine fragrance, we investigated the expression pattern of a putative JMT gene that encodes a jasmonate $\mathrm{O}$-methyl transferase. Indeed, the volatile plant hormone jasmonate and methyl-jasmonate are also directly involved in flower fragrance [28]. As expected, $J M T$ expression appeared to be highly specific in coffee flowers (Table 3).

Finally, the bean-specific expression of genes encoding DGAT (Table 3), the enzyme catalyzing the last step of triglyceride synthesis [29], and Oleosin-2, a structural component of oil bodies [30], provided that we had a good signature of storage lipid accumulation in the coffee bean.

\section{Study of Genes Encoding Main Proteins Stored in Mature Beans}

The storage proteins in the bean constitute the major portion of the proteins found in ripe beans. The expression of these proteins is temporally regulated during the coffee cherry ripening period and is restricted to bean tissues such as cotyledons or endosperm [31]. The coffee storage protein 1 ( $\operatorname{csp} 1$ ) mRNA encoding $11 \mathrm{~S}$ globulin is highly accumulated in ripe beans and poorly detected in leaves or flowers (Table 4) as shown in previous publications $[10,32]$. The coffee storage protein 2 (csp2) mRNA is also detected in the ripe bean but considerably less than $c s p 1$, suggesting that among the $c s p$ gene family a strong difference of expression can be observed from one member to another.

The late embryogenesis abundant (LEA) proteins, a diverse class of highly abundant, heat-stable proteins, 


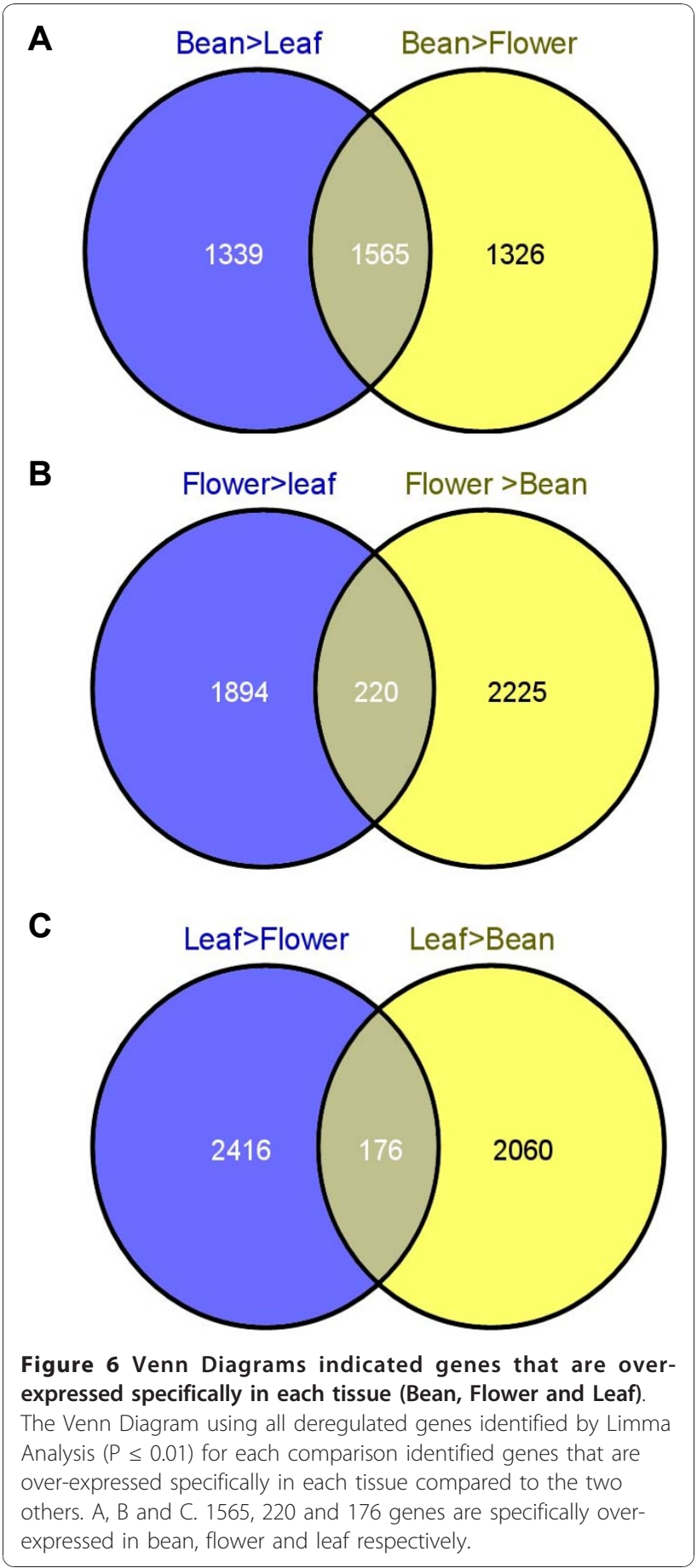

accumulate late in embryo maturation or in endosperm. This accumulation coincides with the acquisition of desiccation tolerance that occurs also during coffee bean ripening. These proteins can be detected in vegetative organs, especially under stress conditions such as cold, drought, or high salinity [33].

CCLEAP2 and CCLEAP3 are highly expressed in ripe beans. While CCLEAP3 is not detected in flowers and leaves (Table 4), CcLEAP2 is significantly expressed in flowers. CCLEAP4 is expressed significantly in the three tissues analyzed but its manifestation in beans is quite low compared to CCLEAP2 and CCLEAP3. This wide difference of expression is also largely observed in Arabidopsis [33] among the 51 LEA proteins identified in the genome, suggesting different functions for each member of this superfamily.

\section{Utility of the 15k Microarray for Different Coffee Species}

Although the long oligonucleotides spotted on the $15 \mathrm{~K}$ coffee microarray were defined from Coffea canephora EST sequences principally derived from genes expressed during coffee fruit development and leaves. We tested the microarray utility tool for two other coffee species, namely $C$. arabica and $C$. eugenioides. In these two species and in C. canephora, evolutionary (divergence) is quite recent ( $<100000$ to 10000 years) and their nucleotide divergence was recently estimated at below 5\% [34]. Furthermore, C. canephora and C. eugenioides are considered to be the diploid parents of $C$. arabica (tetraploïd) [34].

Transcriptomic differences between the two Arabica genotypes (data not shown) appeared minor, so for the present study we considered the average response of both genotypes for the Arabica species. We observed that 8226,8270 and 8530 genes were significantly expressed in comparison to the background noise in C. Canephora, C. Arabica and C. Eugenoides respectively (Additional File 5). These last results indicate that about $53 \%$ of the genes represented on the chip are specifically expressed in leaves. We also calculated that $97.4 \%$ of these expressed genes are common in the three species.

A large difference was observed between the transcriptomes of the parental diploids C. eugenioiddes and C. canephora as $54.5 \%$ of the 15,522 genes were differentially expressed (Figure 5). Among the differentiallyexpressed genes, equivalent proportions were up-regulated in each parent $27.9 \%$ for C. canephora versus $26.6 \%$ for C. eugenioides (Figure 5). A high fraction of genes was differentially expressed between C. arabica and their parents, between 7047 and 8100 genes were indicated as being differentially expressed in C. arabica and C. eugenioïdes and C. canephora respectively. Of these, 23 to $26 \%$ were upregulated in C. arabica and 23-26\% were up-regulated in the two diploid species (respectively C. eugenioides and C. canephora). Finally, a low variation $(\sim 5.4 \%)$ in percentages of differentially expressed genes was observed between the three comparisons.

It can be stated that our microarray tool may be used to analyze global expression not only in Coffea canephora but also in other important species such as Coffea arabica (which represents $70 \%$ of the coffee market) or wild species such as Coffea eugenoïdes. 
Table 3 Expression Patterns of a few Genes Involved in Well-Characterized Lipid Biosynthetic Pathways

\begin{tabular}{|c|c|c|c|c|c|c|c|c|}
\hline Gene & $\begin{array}{c}\text { SGN } \\
\text { Accession }\end{array}$ & Putative Function & $\begin{array}{c}E \\
\text { value }\end{array}$ & $\begin{array}{l}\% \\
\text { ld }\end{array}$ & $\begin{array}{c}\mathrm{B} / \mathrm{L} \\
\text { Ratio }\end{array}$ & $\begin{array}{c}\text { B/F } \\
\text { Ratio }\end{array}$ & $\begin{array}{l}\text { L/F } \\
\text { Ratio }\end{array}$ & $\begin{array}{l}\text { Tissue } \\
\text { Specificity }\end{array}$ \\
\hline$D G A T$ & SGN-U349452 & Acyl-CoA Diacylglycerol acyltransferase (At2g19450) & $1 \mathrm{E}-103$ & 78 & 4 & 8.7 & 2 & Bean $>L>F$ \\
\hline $\begin{array}{l}\text { OLE- } \\
2\end{array}$ & SGN-U350187 & Oleosin CcOLE-2 (AY841272) & 0 & 100 & 9.6 & 9.1 & 1.3 & Bean $>L-F$ \\
\hline FAD8 & SGN-U349395 & Plastidial Linoleate Desaturase FAD8 (At5g05580) & 0 & 73 & 0.021 & 0.085 & 3.8 & Leaf $>F>B$ \\
\hline KCS & SGN-U359520 & -Ketoacyl-CoA Synthase (At1g68530) & $2 \mathrm{E}-87$ & 64 & 0.023 & 0.526 & 21 & Leaf $>F>B$ \\
\hline Fat $B$ & SGN-U350529 & Acyl-ACP Thioesterase (At1g08510) & $1 \mathrm{E}-153$ & 78 & 1.1 & 0.196 & 0.213 & Flower $>$ L-B \\
\hline$J M T$ & SGN-U349158 & $\begin{array}{l}\text { SAM:jasmonic acid carboxyl methyltransferase } \\
\text { (At1G19640) }\end{array}$ & $3 E-34$ & 42 & 4.6 & 0.0025 & 0.0087 & Flower $>$ L-B \\
\hline
\end{tabular}

Comparison: bean to leaves $(\mathrm{B} / \mathrm{L})$, bean to flower $(\mathrm{B} / \mathrm{F})$, leaf to flower $(\mathrm{L} / \mathrm{F})$.

Table 4 Expression Patterns of a few Genes encoding Potential Storage Proteins

\begin{tabular}{|c|c|c|c|c|c|c|c|c|}
\hline Gene & $\begin{array}{l}\text { SGN } \\
\text { accession }\end{array}$ & Putative function & $\begin{array}{c}\mathrm{E} \\
\text { value }\end{array}$ & $\begin{array}{l}\% \\
\text { Id }\end{array}$ & $\begin{array}{l}\mathrm{B} / \mathrm{L} \\
\text { ratio }\end{array}$ & $\begin{array}{l}\mathrm{B} / \mathrm{F} \\
\text { ratio }\end{array}$ & $\begin{array}{l}\mathrm{L} / \mathrm{F} \\
\text { ratio }\end{array}$ & $\begin{array}{l}\text { Tissue } \\
\text { specificity }\end{array}$ \\
\hline $\operatorname{csp} 1$ & SGN-U350946 & $\begin{array}{l}11 \text { S plant bean storage protein Coffea arabica } \\
\text { (Y16975) }\end{array}$ & $1-e 134$ & 100 & 103 & 93 & 0.925 & $B>F-L$ \\
\hline $\operatorname{csp} 2$ & SGN-U347807 & 11 S plant bean storage protein (At2G28490) & $1 e-121$ & 50 & 28.56 & 12.75 & 0.315 & $B>F>L$ \\
\hline CCLEAP2 & SGN-U350577 & Late embryogenesis abundant protein (At1G52690) & $1 e-12$ & 60 & 257 & 6.97 & 0.01 & $B>F>L$ \\
\hline CCLEAP3 & SGN-348605 & Late embryogenesis abundant protein (At2G40170) & $8 e-24$ & 73 & 592.76 & 339.53 & 0.38 & $B>F-L$ \\
\hline CCLEAP4 & SGN-347291 & Late embryogenesis abundant protein (At4g02380) & $2 \mathrm{e}-15$ & 50 & 6.26 & 1.82 & 0.32 & $B>F>L$ \\
\hline
\end{tabular}

Comparison: bean to leaves (B/L), bean to flower (B/F), leaf to flower (L/F); csp (coffee storage protein); LEAP (late embryogenesis abundant protein).

\section{Conclusions}

We present here the creation and validation of the first coffee oligonucleotide-based microarray tool for functional genomic studies in coffee. Our results reveal that this new tool applies to high-throughput gene expression analyses in various Coffea species. Furthermore, the use of the array has proven to be valid for genomic studies on different plant tissues. As proof of principle, we have reported changes in gene expression generated by this microarray in two independent experiments. The statistical analyses of our microarray data, the correct correlation between QPCR and the microarray data validate our chip. Overall the coffee microarray (designated as "PUCE CAFE") offers the possibility to carry out functional genomic studies in a wide variety of research areas such as plant development, biotic and abiotic stress response or fruit quality traits. This new tool will be valuable for researchers interested in Coffea transcriptomics and will be available through the MGX platform.

\section{Additional material}

Additional file 1: The Missing Genes. List of 195 unigenes for which the design of specific oligonucleotides could not be achieved and that are therefore absent from the PUCE CAFE microarray.

Additional file 2: Oligonucleotides that may cross-hybridize with several sequences. List of 371 oligonucleotides spotted on the microarray which have $>70 \%$ of overall identity to another unigene and have a contiguous identical length of more than $20 \mathrm{nt}$ common to another unigene.
Additional file 3: Q-PCR and Microarray Data for 111 Genes of Interest Extracted from Experiment 1. Microarray and Q-PCR were compared by calculating the Pearson correlation coefficients. For the microarray, the data input into the correlation analysis was the Log2 ratio value of the weighted average and for each gene on the composite array representing all replicates. For qRT-PCR, we used the mean Log2 ratio value representing all replicate plants. All correlation analyses carried an alpha value of 0.01 and were performed using SAS 9.2. The list of the primers and relative sequence used for the GPCR are indicted as well as the efficiency of amplification for each couple of primers. Additional file 4: MIQE document (Minimum Information for Publication of Quantitative Real-Time PCR Experiments).

Additional file 5: Genes Significantly Expressed in Experiment 2. For each species analyzed in Experiment 2 (C. arabica, C. canephora, C. eugenoides), genes showing a significant hybridization signal compared to the background noise are indicated.

Additional file 6: Gene Ontology Functional Enrichment. Functional enrichment using gene ontology terms and Fisher's Exact Test was performed using Blast2 $\mathrm{GO}$ for the sets of up-regulated unigenes in each of the three tissues (bean, leaf and flower). The full set of unigenes of the PUCE CAFE array was used as the reference set. The resulting $\mathrm{p}$ values are indicated after adjusting for FDR multiple testing results. The over- or under-expressed functions at $\mathrm{p} \leq 0.01$ are specified.

Additional file 7: Reproducibility of Replicates for the Leaf Tissue in the Leaf-Flower Comparison. Coefficient of variation (CV\%) for all cDNAs spotted on the array based on raw data mean fluorescence values plotted against the relative rank of the CV. CVs were estimated from raw data derived from six replicates (i.e. 2 dye $\times 3$ biological replicates). For this tissue and for this experiment, $90 \%$ of the spots on the arrays could be determined with a CV of less than 35\%. For the other experiments, $90 \%$ of the spots on the arrays could be determined with CV between 32 and 42\%.

Additional file 8: Theoretical Power Analysis for a Two-sample Ttest to Detect a 1.25 to 2.50 Fold Change in Gene Expression as a Function of CV\%. Indicated sample size $=6$ and a Type I error (false positive rate) of 0.1 were used as input values to determine the fraction of changes in gene expression that would be detected at a given CV\%. 
A power of 1.0 denotes a Type II error (false negative rate) of zero, i.e. $100 \%$ of all changes that occurred were detected.

Additional file 9: Lists of Genes Specifically Over-expressed in each Tissue Compared to the Two Others (Bean, Flower and Leaf). The first 3 datasheets correspond to the lists of genes differentially expressed for each comparison identified by the Limma Analysis $(p=0.01)$. The last datasheet corresponds to the lists of genes specifically over-expressed in each tissue compared to the two others.

\section{Acknowledgements}

This work was completed thanks to a grant from Génoplante, the French consortium for plant genomics. We would like to thank Maud Lepelley and Philippe Schaeffer.

\section{Author details}

'Nestlé R\&D Tours, 101 Avenue Gustave Eiffel, Notre Dame d'Oé, BP 49716, 37097 Tours Cedex 2, France. ${ }^{2}$ Montpellier Genomix, IGF, 141 rue de la Cardonille, 34094 Montpellier Cedex 05, France. ${ }^{3}$ Boyce Thompson Institute for Plant Research, Tower Road, Ithaca, New York 14853-1801, USA. ${ }^{4}$ IRDCIRAD, UMR RPB, BP 64501, 34394 Montpellier, France. ${ }^{5}$ IRD, UMR DIAPC, BP 64501, 34394 Montpellier, France. ${ }^{6}$ CIRAD avenue Agropolis, 34398 Montpellier Cedex 5, France.

\section{Authors' contributions}

IP and BB have contributed equally to this work. The project was coordinated by $\mathrm{P}, \mathrm{PL}$ and $\mathrm{BB}$. All the authors have read the manuscript and agree with the contents.

\section{Competing interests}

The authors declare that they have no competing interests.

Received: 24 June 2010 Accepted: 5 January 2011

Published: 5 January 2011

\section{References}

1. Aharoni A, Vorst O: DNA microarrays for functional plant genomics. Plant Mol Biol 2002, 48:99-118.

2. Galbraith DW: DNA microarray analyses in higher plants. OMICS 2006, 10:455-473.

3. Clarke JD, Zhu T: Microarray analysis of the transcriptome as a stepping stone towards understanding biological systems: practical considerations and perspectives. Plant J 2006, 45:630-650.

4. Mascarell-Creus A, Canizares J, Vilarrasa-Blasi J, Mora-Garcia S, Blanca J, Gonzalez-Ibeas D, Saladie M, Roig C, Deleu W, Pico-Silvent B, Lopez-Bigas N, Aranda MA, Garcia-Mas J, Nuez F, Puigdomenech P, Cano-Delgado Al: An oligo-based microarray offers novel transcriptomic approaches for the analysis of pathogen resistance and fruit quality traits in melon (Cucumis melo L.). BMC Genomics 2009, 10:467.

5. Martinez-Godoy MA, Mauri N, Juarez J, Marques MC, Santiago J, Forment J, Gadea J: A genome-wide $20 \mathrm{~K}$ citrus microarray for gene expression analysis. BMC Genomics 2008, 9:318

6. Davis AP, Govaerts R, Bridson DM, Stoffelen P: An annotated taxonomic conspectus of the genus Coffea (Rubiaceae). Botanical Journal of the Linnean Society 2006, 152:165-512.

7. Pendergrast M: Coffee second only to oil? Is coffee really the second largest commodity? Tea and Coffe Trade Journal 2009, 181:38-41.

8. Lin C, Mueller LA, MC CJ, Crouzillat D, Petiard V, Tanksley SD: Coffee and tomato share common gene repertoires as revealed by deep sequencing of seed and cherry transcripts. Theor Appl Genet 2005, 112:114-130.

9. Poncet V, Rondeau M, Tranchant C, Cayrel A, Hamon S, de KA, Hamon P: SSR mining in coffee tree EST databases: potential use of EST-SSRs as markers for the Coffea genus. Mol Genet Genomics 2006, 276:436-449.

10. Joët T, Laffargue A, Salmona J, Doulbeau S, Descroix F, Bertrand B, de Kochko A, Dussert S: Metabolic pathways in tropical dicotyledonous albuminous seeds: Coffea arabica as a case study. New Phytol 2009, 182:146-162.
11. Ewing B, Hillier L, Wendl MC, Green P: Base-calling of automated sequencer traces using phred. I. Accuracy assessment. Genome Res 1998, 8:175-185

12. Altschul SF, Gish W, Miller W, Myers EW, Lipman DJ: Basic local alignment search tool. J Mol Biol 1990, 215:403-410.

13. Huang $X$, Madan A: CAP3: A DNA sequence assembly program. Genome Res 1999, 9:868-877.

14. Iseli C, Jongeneel CV, Bucher P: ESTScan: a program for detecting, evaluating, and reconstructing potential coding regions in EST sequences. Proc Int Conf Intell Syst Mol Biol 1999, 138-148.

15. Hunter S, Apweiler R, Attwood TK, Bairoch A, Bateman A, Binns D, Bork P, Das U, Daugherty L, Duquenne L, Finn RD, Gough J, Haft D, Hulo N, Kahn D, Kelly E, Laugraud A, Letunic I, Lonsdale D, Lopez R, Madera M, Maslen J, McAnulla C, McDowall J, Mistry J, Mitchell A, Mulder N, Natale D, Orengo C, Quinn AF, Selengut JD, Sigrist CJ, Thimma M, Thomas PD, Valentin F, Wilson $D$, Wu CH, Yeats C: InterPro: the integrative protein signature database. Nucleic Acids Res 2009, 37:D211-D215.

16. Vandesompele J, De Preter K, Pattyn F, Poppe B, Van Roy N, De Paepe A, Speleman F: Accurate normalization of real-time quantitative RT-PCR data by geometric averaging of multiple internal control genes. Genome Biol 2002, 3:RESEARCH0034

17. Wettenhall JM, Smyth GK: LimmaGUI: a graphical user interface for linear modeling of microarray data. Bioinformatics 2004, 20:3705-3706.

18. Tusher VG, Tibshirani R, Chu G: Significance analysis of microarrays applied to the ionizing radiation response. Proc Natl Acad Sci USA 2001, 98:5116-5121.

19. Benjamini $Y$, Hochberg $Y$ : Controlling the false discovery rate: a practical and powerful approach to multiple testing. J Roy Statist Soc Ser 1995, B 57:289-300.

20. Troein C, Vallon-Christersson J, Saal LH: An introduction to BioArray Software Environment. Methods Enzymol 2006, 411:99-119.

21. Gotz S, Garcia-Gomez JM, Terol J, Williams TD, Nagaraj SH, Nueda MJ, Robles M, Talon M, Dopazo J, Conesa A: High-throughput functional annotation and data mining with the Blast2GO suite. Nucleic Acids Res 2008, 36:3420-3435.

22. Degenkolbe T, Hannah MA, Freund S, Hincha DK, Heyer AG, Kohl KI: A quality-controlled microarray method for gene expression profiling. Anal Biochem 2005, 346:217-224.

23. Yuen T, Wurmbach E, Pfeffer RL, Ebersole BJ, Sealfon SC: Accuracy and calibration of commercial oligonucleotide and custom CDNA microarrays. Nucleic Acids Res 2002, 30:e48.

24. Morey JS, Ryan JC, Van Dolah FM: Microarray validation: factors influencing correlation between oligonucleotide microarrays and realtime PCR. Biol Proced Online 2006, 8:175-193.

25. Campos PS, Quartin V, Ramalho JC, Nunes MA: Electrolyte leakage and lipid degradation account for cold sensitivity in leaves of Coffea sp. plants. J Plant Physiol 2003, 160:283-292.

26. Nikolova-Damyanova B, Velikova R, Jham GN: Lipid classes, Fatty Acid Composition and Triacylglycerol Molecular Species in Crude Coffee Beans Harvested in Brazil. Food research international 1998, 31:479-486.

27. Dormann $P$, Voelker TA, Ohlrogge JB: Accumulation of palmitate in Arabidopsis mediated by the acyl-acyl carrier protein thioesterase FATB1. Plant Physiol 2000, 123:637-644.

28. Cheong JJ, Choi YD: Methyl jasmonate as a vital substance in plants. Trends Genet 2003, 19:409-413.

29. Beaudoin F, Napier JA: Biosynthesis and compartmentation of triacylglycerol in higher plants. In Lipid Metabolism and Membrane Biogenesis (Topics in Current Genetics, Vol. 6). Edited by: Daum G. Berlin: Springer-Verlag; 2004:267-287.

30. Simkin AJ, Qian T, Caillet V, Michoux F, Ben AM, Lin C, Tanksley S, McCarthy J: Oleosin gene family of Coffea canephora: quantitative expression analysis of five oleosin genes in developing and germinating coffee grain. J Plant Physiol 2006, 163:691-708.

31. Shewry PR, Napier JA, Tatham AS: Seed storage proteins: structures and biosynthesis. Plant Cell 1995, 7:945-956.

32. Marraccini P, Deshayes A, Pétiard V, Rogers WJ: Molecular cloning of the complete $11 \mathrm{~S}$ seed storage protein gene of Coffea arabica and promoter analysis in transgenic tobacco plants. Plant Physiology and Biochemistry 1999, 37:273-282. 
33. Hundertmark M, Hincha DK: LEA (Late Embryogenesis Abundant) Proteins and their Encoding Genes in Arabidopsis Thaliana. BMC Genomics 2008, 9:118.

34. Lashermes P, Combes MC, Robert J, Trouslot P, D'Hont A, Anthony F, Charrier A: Molecular characterisation and origin of the Coffea arabica L. genome. Mol Gen Genet 1999, 261:259-266.

\section{doi:10.1186/1471-2164-12-5}

Cite this article as: Privat et al: The 'PUCE CAFE' Project: the First $15 \mathrm{~K}$

Coffee Microarray, a New Tool for Discovering Candidate Genes

correlated to Agronomic and Quality Traits. BMC Genomics 2011 12:5.

Submit your next manuscript to BioMed Central and take full advantage of:

- Convenient online submission

- Thorough peer review

- No space constraints or color figure charges

- Immediate publication on acceptance

- Inclusion in PubMed, CAS, Scopus and Google Scholar

- Research which is freely available for redistribution

Submit your manuscript at www.biomedcentral.com/submit 